\title{
Fixed Point Theorems for Fuzzy Mappings on Closed Subset of Hilbert Spaces for Rational Expression
}

\author{
Buthainah A. A. Ahmed ${ }^{1}$, Manar Falih Dheyab ${ }^{2}$ \\ Department of Mathematics, College of Science, University of Baghdad, Iraq
}

\begin{abstract}
The aim of this paper is to obtain a common fixed point for fuzzy mapping on closed subset of Hilbert spaces for rational expression. The proof rely on the parallelogram law in Hilbert spaces.
\end{abstract}

Keywords: fuzzy mapping, fixed point, approximate quantity and Hilbert space

\section{Introduction}

The concept of fuzzy set was introduce by L.Zadeh [2]in 1965.After that a lot of work has been done regarding fuzzy set and fuzzy mappings. The concept of fuzzy mapping was first introduced by Heilpern [3], he proved fixed point theorem for fuzzy contraction mappings which is a fuzzy analogue of the fixed point theorem for multy valued mapping of Nadler[4]. In 2015 [1] Jagdish C. Chaudhary, Jatin, Patel and Chirag proved some fixed point theorems in fuzzy mappings containing the rational expressions. In this paper we are proving some fixed point for fuzzy mapping on closed subset of Hilbert spaces for rational expression.

\section{Preliminaries}

In the following discussions mainly follow the definition and notions due to Helipern [3]. Let $\mathrm{H}$ be a Hilbert space and $\mathrm{F}(\mathrm{H})$ be a collection of all fuzzy sets in $H$. Let $A \in F(H)$ and $\alpha \in[0,1]$ the $\alpha-$ level set of $A$, denoted by $\mathrm{A}_{\alpha}$ is defined by

$$
\begin{gathered}
A_{\alpha}=\{x: A(x) \geq \alpha\} \text { if } \alpha \in[0,1] \\
A_{0}=\overline{\{x: A(x)>\alpha\}}
\end{gathered}
$$

Where $\overline{\mathrm{B}}$ denotes the closure of a set $\mathrm{B}$.

Definition 2.1[3]: A fuzzy set $A$ is said to be an approximate quantity if and only if $\mathrm{A}_{\alpha}$ is compact and convex for each $\alpha \in[0,1]$, and $\sup _{\mathrm{x} \in \mathrm{X}} \mathrm{A}(\mathrm{x})=1$. When $\mathrm{A}$ is an approximate quantity and $\mathrm{A}\left(\mathrm{x}_{0}\right)=1$ for $\operatorname{somex}_{0} \in \mathrm{H}, \mathrm{A}$ is identified with an approximate of $x_{0}$.

The collection of all fuzzy sets in $\mathrm{H}$ is denoted by $\mathrm{F}(\mathrm{H})$ and $\mathrm{W}(\mathrm{H})$ is the sub collection of all approximate quantities.

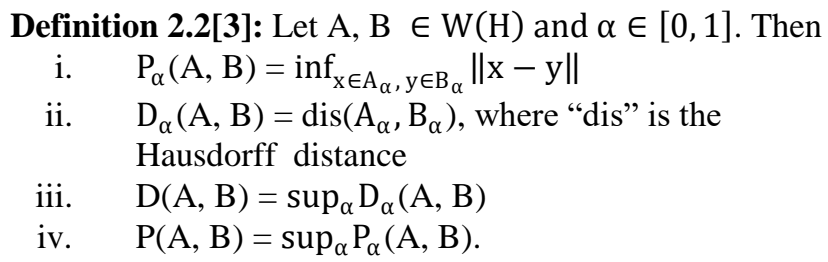

It is to be noted that for any ' $\alpha$ ', $\mathrm{P}_{\alpha}$ is a non decreasing as well as continuous function.

Definition 2.3[2]. Let $A, B \in W(H)$. An approximate quantity $A$ is said to be more accurate than $B$ (denoted by $A \subset B)$ if and only if $A(x) \leq B(x), \forall x \in H$.
Definition 2.4[3]: A mapping $\mathrm{T}$ from the set $\mathrm{H}$ into $\mathrm{W}(\mathrm{H})$ is said to be fuzzy mapping.

Definition 2.5[3]: The point $x \in H$ is called fixed point for the fuzzy mapping $\mathrm{T}$ if and only if $\{\mathrm{x}\} \subset \mathrm{T}(\mathrm{x})$. We shall use the following lemmas due to Helipern.

Lemma 2.6[3]: $P_{\alpha}(x, B) \leq\|x-y\|+P_{\alpha}(y, B), \forall x, y \in H$.

Lemma 2.7[3]: If $\left\{\mathrm{x}_{0}\right\} \subset \mathrm{A}$, then $\mathrm{P}_{\alpha}\left(\mathrm{x}_{0}\right.$,

$\leq \mathrm{D}_{\alpha}(\mathrm{A}, \mathrm{B}), \forall \mathrm{B} \in \mathrm{W}(\mathrm{H})$.

Lemma 2.8[3]: $\quad$ Let $A \in W(H)$ and $x_{0} \in H$, if $\left\{\mathrm{x}_{0}\right\} \subset$ A then $\mathrm{P} \alpha(\mathrm{x} 0, \mathrm{~A})=0$, for each $\alpha \in 0,1$.

Lemma 2.9[3]: Let $\mathrm{H}$ be a Hilbert space and $\mathrm{T}$ fuzzy mapping from $\mathrm{H}$ into $\mathrm{W}(\mathrm{H})$ and $\mathrm{x}_{0} \in \mathrm{H}$, then there exist $\mathrm{x}_{1} \in \mathrm{H}$ such that $\left\{\mathrm{x}_{1}\right\} \subset \mathrm{T}\left(\mathrm{x}_{0}\right)$.

\section{Main Results}

The proof rely on the parallelogram law in Hilbert spaces.

Theorem 3.1: Let $\mathrm{C}$ be a closed subset of a Hilbert space $\mathrm{H}$ . Let $\mathrm{T}, \mathrm{S}$ be a fuzzy mapping from $\mathrm{C}$ into $\mathrm{W}(\mathrm{C})$ satisfying : $\mathrm{D}^{2}(\mathrm{~T}(\mathrm{x}), \mathrm{S}(\mathrm{y}))$

$\leq \alpha \mathrm{P}_{\alpha}^{2}(\mathrm{x}, \mathrm{T}(\mathrm{x}))$

$+\beta \mathrm{P}_{\alpha}^{2}(\mathrm{y}, \mathrm{S}(\mathrm{y}))+\gamma \| \mathrm{x}$

$-\mathrm{y} \|^{2}+\delta \min \left\{\mathrm{P}_{\alpha}^{2}(\mathrm{x}, \mathrm{S}(\mathrm{y})), \mathrm{P}_{\alpha}^{2}(\mathrm{y}, \mathrm{T}(\mathrm{x}))\right\}+\mathrm{m}(\mathrm{x}, \mathrm{y})$

For all $\mathrm{x}, \mathrm{y} \in \mathrm{Cwhere} \gamma, \alpha, \beta, \delta, \sigma, \varepsilon \geq 0$ with

$$
\begin{gathered}
\alpha+\beta+\varepsilon+\gamma+\delta+\sigma<1 \text { and } \\
\mathrm{m}(\mathrm{x}, \mathrm{y})=\sigma \frac{\mathrm{P}_{\alpha}^{2}(\mathrm{y}, \mathrm{S}(\mathrm{y}))}{1+\mathrm{P}_{\alpha}^{2}(\mathrm{x}, \mathrm{S}(\mathrm{x})) \mathrm{P}_{\alpha}^{2}(\mathrm{x}, \mathrm{T}(\mathrm{y}))} \\
\quad+\varepsilon \frac{\mathrm{P}_{\alpha}^{2}(\mathrm{x}, \mathrm{S}(\mathrm{x}))}{1+\mathrm{P}_{\alpha}^{2}(\mathrm{y}, \mathrm{S}(\mathrm{x})) \mathrm{P}_{\alpha}^{2}(\mathrm{x}, \mathrm{T}(\mathrm{y}))} .
\end{gathered}
$$

Then $\mathrm{T}$ and $\mathrm{S}$ have a fixed point $\mathrm{z} \in \mathrm{C}$ such that $\{\mathrm{z}\} \subset \mathrm{T}(\mathrm{z}) \cap$ $\mathrm{S}(\mathrm{z})$.

Proof: Let $\mathrm{x}_{0} \in \mathrm{C}$, we construct the sequence $\left\{\mathrm{x}_{\mathrm{n}}\right\} \in \mathrm{C}$ as follows $\left\{\mathrm{x}_{1}\right\} \subset \mathrm{T}\left(\mathrm{x}_{0}\right),\left\{\mathrm{x}_{2}\right\} \subset \mathrm{S}\left(\mathrm{x}_{1}\right)$,

$\left\{x_{2 n+1}\right\} \subset T\left(x_{2 n}\right),\left\{x_{2 n+2}\right\} \subset S\left(x_{2 n+1}\right)$ and $\| x_{2 n}-$ $\mathrm{x} 2 \mathrm{n}+12 \leq \mathrm{D} 12 \mathrm{~T}(\mathrm{x} 2 \mathrm{n}-1), \mathrm{S}(\mathrm{x} 2 \mathrm{n})$.

Now

$\left\|x_{2 n+1}-x_{2 n}\right\|^{2} \leq D_{1}^{2}\left(S\left(x_{2 n-1}\right), T\left(x_{2 n}\right)\right)$, for all $n \in N$. 


\section{International Journal of Science and Research (IJSR) \\ ISSN (Online): 2319-7064}

Index Copernicus Value (2015): 78.96 | Impact Factor (2015): 6.391

$$
\begin{gathered}
\left\|x_{2 n+1}-x_{2 n}\right\|^{2} \leq D^{2}\left(S\left(x_{2 n-1}\right), T\left(x_{2 n}\right)\right) \\
\leq \alpha P_{\alpha}^{2}\left(x_{2 n-1}, S\left(x_{2 n-1}\right)\right) \\
+\beta P_{\alpha}^{2}\left(x_{2 n}, T\left(x_{2 n}\right)\right)+\gamma\left\|x_{2 n}-x_{2 n-1}\right\|^{2} \\
+\delta \min \left\{P_{\alpha}^{2}\left(x_{2 n}, S\left(x_{2 n-1}\right)\right), P_{\alpha}^{2}\left(x_{2 n-1}, T\left(x_{2 n}\right)\right)\right\} \\
+\sigma \frac{P_{\alpha}^{2}\left(x_{2 n}, S\left(x_{2 n-1}\right)\right)}{1+P_{\alpha}^{2}\left(x_{2 n}, T\left(x_{2 n}\right)\right) P_{\alpha}^{2}\left(x_{2 n}, S\left(x_{2 n-1}\right)\right)} \\
+\varepsilon \frac{P_{\alpha}^{2}\left(x_{2 n-1}, S\left(x_{2 n-1}\right)\right)}{1+P_{\alpha}^{2}\left(x_{2 n}, T\left(x_{2 n}\right)\right) P_{\alpha}^{2}\left(x_{2 n}, S\left(x_{2 n-1}\right)\right)} \\
\leq \alpha\left\|x_{2 n}-x_{2 n-1}\right\|^{2} \quad+\beta\left\|x_{2 n}-x_{2 n+1}\right\|^{2}+\gamma\left\|x_{2 n}-x_{2 n-1}\right\|^{2} \\
+\delta \min \left\{\left\|x_{2 n}-x_{2 n}\right\|^{2},\left\|x_{2 n+1}-x_{2 n-1}\right\|^{2}\right\} \\
+\sigma \frac{\left\|x_{2 n}-x_{2 n}\right\|^{2}}{1+\left\|x_{2 n}-x_{2 n+1}\right\|^{2}\left\|x_{2 n}-x_{2 n}\right\|^{2}} \\
\quad+\varepsilon \frac{\left\|x_{2 n}-x_{2 n-1}\right\|^{2}}{1+\left\|x_{2 n}-x_{2 n+1}\right\|^{2}\left\|x_{2 n}-x_{2 n}\right\|^{2}} \\
(1-\beta)\left\|x_{2 n+1}-x_{2 n}\right\|^{2} \leq(\alpha+\gamma+\varepsilon)\left\|x_{2 n-1}-x_{2 n}\right\|^{2} \\
\left\|x_{2 n+1}-x_{2 n}\right\|^{2} \leq \frac{\alpha+\gamma+\varepsilon}{1-\beta}\left\|x_{2 n-1}-x_{2 n}\right\|^{2} \\
\text { Putting } q=\frac{\alpha+\gamma+\varepsilon}{1-\beta}<1 \\
\text { Then } 1
\end{gathered}
$$

Then, we have

$$
\left\|x_{2 n+1}-x_{2 n}\right\|^{2} \leq q\left\|x_{2 n-1}-x_{2 n}\right\|^{2}
$$

Now, for any positive integer $p$

$$
\begin{gathered}
\left\|x_{n}-x_{n+p}\right\|^{2} \leq q\left\|x_{n}-x_{n+1}\right\|^{2} \\
+\left\|x_{n+1}-x_{n+2}\right\|^{2}+.+\left\|x_{n+p-1}-x_{n+p}\right\|^{2} \\
\leq\left(q^{n}+q^{n+1}+q^{n+2}+\cdots+q^{n+p-1}\left\|x_{1}-x_{0}\right\|^{2}\right. \\
\leq \frac{q^{n}}{1-q}\left\|x_{1}-x_{0}\right\|^{2}
\end{gathered}
$$

Which implies that $\left\|x_{n}-x_{n+p}\right\|^{2} \rightarrow 0$ as $n \rightarrow \infty$

Hence

\section{\{}

$\left.x_{n}\right\}$ is cauchy sequance in $H$, but $H$ is a Hilbert space, so $\left\{x_{n}\right\}$ is

converge to $\mathrm{z}$ and since $C$ is closed and $\left\{x_{n}\right\}$ is sequence in C

Then $z \in C$, such that $\lim _{n \rightarrow \infty} x_{n}=z$

$P_{\alpha}^{2}(z, T(z)) \leq\left\|z-x_{2 n}\right\|^{2}+P_{\alpha}^{2}\left(x_{2 n}, T(z)\right)$

$$
\begin{aligned}
& \leq\left\|z-x_{2 n}\right\|^{2}+D_{\alpha}^{2}\left(S\left(x_{2 n-1}\right), T(z)\right) \\
& \leq\left\|z-x_{2 n}\right\|^{2}+D^{2}\left(S\left(x_{2 n-1}\right), T(z)\right)
\end{aligned}
$$

$\leq\left\|z-x_{2 n}\right\|^{2}+\alpha P_{\alpha}^{2}\left(x_{2 n-1}, S\left(x_{2 n-1}\right)\right)+\beta P_{\alpha}^{2}(z, T(z))$

$+\gamma \| x_{2 n-1}$

$-z \|^{2}+\delta \min \left\{P_{\alpha}^{2}\left(x_{2 n-1}, T(z)\right), P_{\alpha}^{2}\left(z, S\left(x_{2 n-1}\right)\right)\right\}$

$+\sigma \frac{P_{\alpha}^{2}\left(z, S\left(x_{2 n-1}\right)\right)}{1+P_{\alpha}^{2}\left(x_{2 n-1}, S\left(x_{2 n-1}\right)\right) P_{\alpha}^{2}\left(x_{2 n-1}, T(z)\right)}$

$$
+\varepsilon \frac{P_{\alpha}^{2}\left(x_{2 n-1}, S\left(x_{2 n-1}\right)\right)}{1+P_{\alpha}^{2}\left(z, S\left(x_{2 n-1}\right)\right) P_{\alpha}^{2}\left(x_{2 n-1}, T(z)\right)}
$$

$\leq\left\|z-x_{2 n}\right\|^{2}+\alpha\left\|x_{2 n-1}-x_{2 n}\right\|^{2}$

$$
+\beta P_{\alpha}^{2}(z, T(z))+\gamma\left\|x_{2 n-1}-z\right\|^{2}
$$

$+\delta \min \left\{P_{\alpha}^{2}\left(x_{2 n-1}, T(z)\right),\left\|x_{2 n}-z\right\|^{2}\right\}$

$$
+\sigma \frac{\left\|z-x_{2 n}\right\|^{2}}{1+\left\|x_{2 n}-x_{2 n-1}\right\|^{2} P_{\alpha}^{2}\left(x_{2 n-1}, T(z)\right)}
$$$$
+\varepsilon \frac{\left\|x_{2 n}-x_{2 n-1}\right\|^{2}}{1+\left\|x_{2 n}-z\right\|^{2} P_{\alpha}^{2}\left(x_{2 n-1}, T(z)\right)}
$$

$P_{\alpha}^{2}(z, T(z)) \leq \alpha\left\|x_{2 n-1}-x_{2 n}\right\|^{2}+\beta P_{\alpha}^{2}(z, T(z))$

$$
+\varepsilon\left\|x_{2 n}-x_{2 n-1}\right\|^{2}
$$

$(1-\beta) P_{\alpha}^{2}(z, T(z)) \leq(\alpha+\varepsilon)\left\|x_{2 n}-x_{2 n-1}\right\|^{2}$

$$
P_{\alpha}^{2}(z, T(z)) \leq \frac{\alpha++\varepsilon}{1-\beta}\left\|x_{2 n-1}-x_{2 n}\right\|^{2}
$$

Putting $q=\frac{\alpha+\varepsilon}{1-\beta}<1$

Then, we have

$$
P_{\alpha}^{2}(z, T(z)) \leq q\left\|x_{2 n-1}-x_{2 n}\right\|^{2} \leq q^{n}\left\|x_{2 n-1}-x_{2 n}\right\|^{2}
$$

As $\rightarrow \infty$, then $P_{\alpha}^{2}(z, T(z))=0$

Hence we get $\{z\} \subset T(z)$.

Similarly, $\{z\} \subset S(z)$.

Theorem 3.2: Let $\mathrm{C}$ be a closed subset of a Hilbert space $\mathrm{H}$

. Let $\mathrm{T}, \mathrm{S}$ be a fuzzy mapping from $\mathrm{C}$ into $\mathrm{W}(\mathrm{C})$ satisfying

$$
\begin{aligned}
& D^{2}(S(x), T(y)) \\
\leq & \alpha \frac{P_{\alpha}^{2}(y, T(x))+P_{\alpha}^{2}(x, S(x))+P_{\alpha}^{2}(y, S(x))}{1+P_{\alpha}^{2}(y, S(x)) P_{\alpha}^{2}(x, T(y))}+ \\
& \quad \beta\left[P_{\alpha}^{2}(y, T(y))+P_{\alpha}^{2}(y, S(x))\right]+\gamma\|x-y\|^{2}
\end{aligned}
$$

For all $\mathrm{x}, \mathrm{y} \in \mathrm{C}$ where $\gamma, \alpha, \beta \geq 0$ with

$2 \alpha+\beta+\gamma<1$. Then there is $\mathrm{z} \in \mathrm{C}$ such that $\mathrm{z}$ is a common fixed point for $\mathrm{T}$ and $\mathrm{S}$.

Proof: Let $x_{0} \in C$, we construct the sequence $\left\{x_{n}\right\} \in C$ as follows $\left\{\mathrm{x}_{1}\right\} \subset \mathrm{T}\left(\mathrm{x}_{0}\right),\left\{\mathrm{x}_{2}\right\} \subset \mathrm{S}\left(\mathrm{x}_{1}\right)$,

$\left\{x_{2 n+1}\right\} \subset T\left(x_{2 n}\right),\left\{x_{2 n+2}\right\} \subset S\left(x_{2 n+1}\right)$ and $\| x_{2 n}-$ $x 2 n+12 \leq D 12 T(x 2 n-1), S(x 2 n)$.

Now

$\left\|x_{2 n+1}-x_{2 n}\right\|^{2} \leq D_{1}^{2}\left(S\left(x_{2 n-1}\right), T\left(x_{2 n}\right)\right)$, for all $n \in N$.

$\left\|x_{2 n+1}-x_{2 n}\right\|^{2} \leq D^{2}\left(S\left(x_{2 n-1}\right), T\left(x_{2 n}\right)\right)$

$$
\begin{aligned}
& \leq \alpha \frac{P_{\alpha}^{2}\left(x_{2 n}, T\left(x_{2 n}\right)\right)+P_{\alpha}^{2}\left(x_{2 n-1}, S\left(x_{2 n-1}\right)\right)+P_{\alpha}^{2}\left(x_{2 n}, S\left(x_{2 n-1}\right)\right)}{1+P_{\alpha}^{2}\left(x_{2 n}, S\left(x_{2 n-1}\right)\right) P_{\alpha}^{2}\left(x_{2 n-1}, T\left(x_{2 n}\right)\right)} \\
& +\quad \beta\left[P_{\alpha}^{2}\left(x_{2 n}, T\left(x_{2 n}\right)\right)+P_{\alpha}^{2}\left(x_{2 n}, S\left(x_{2 n-1}\right)\right)\right] \\
& \quad+\gamma\left\|x_{2 n-1}-x_{2 n}\right\|^{2}
\end{aligned}
$$

$+\beta\left[\left\|x_{2 n-1}-x_{2 n}\right\|^{2}+\left\|x_{2 n}-x_{2 n}\right\|^{2}\right]+\gamma\left\|x_{2 n-1}-x_{2 n}\right\|^{2}$

Then $\left\|x_{2 n+1}-x_{2 n}\right\|^{2} \leq(2 \alpha+\beta+\gamma)\left\|x_{2 n-1}-x_{2 n}\right\|^{2}$

Putting $q=2 \alpha+\beta+\gamma<1$

Then, we have

$$
\left\|x_{2 n+1}-x_{2 n}\right\|^{2} \leq q\left\|x_{2 n-1}-x_{2 n}\right\|^{2}
$$

Now, for any positive integer $p$

$$
\begin{aligned}
&\left\|x_{n}-x_{n+p}\right\|^{2} \leq q\left\|x_{n}-x_{n+1}\right\|^{2} \\
&+\left\|x_{n+1}-x_{n+2}\right\|^{2}+\ldots+\| x_{n+p-1} \\
&-x_{n+p} \|^{2} \\
& \leq\left(q^{n}+q^{n+1}+q^{n+2}+\cdots+q^{n+p-1}\left\|x_{1}-x_{0}\right\|^{2}\right. \\
& \leq \frac{q^{n}}{1-q}\left\|x_{1}-x_{0}\right\|^{2}
\end{aligned}
$$

Which implies that $\left\|x_{n}-x_{n+p}\right\|^{2} \rightarrow 0$ as $n \rightarrow \infty$

Hence,

$\left\{x_{n}\right\}$ is cauchy sequance in $H$, but $H$ is a Hilbert space. so $\left\{x_{n}\right\}$ is converge to $z$ and since $C$ is closed and $\left\{x_{n}\right\}$ is sequence in $C$.

Then $z \in C$, such that $\lim _{n \rightarrow \infty} x_{n}=z$

$$
\begin{aligned}
P_{\alpha}^{2}(z, T(z)) & \leq\left\|z-x_{2 n}\right\|^{2}+P_{\alpha}^{2}\left(x_{2 n}, T(z)\right) \\
& \leq\left\|z-x_{2 n}\right\|^{2}+D_{\alpha}^{2}\left(S\left(x_{2 n-1}\right), T(z)\right) \\
& \leq\left\|z-x_{2 n}\right\|^{2}+D^{2}\left(S\left(x_{2 n-1}\right), T(z)\right)
\end{aligned}
$$

\section{Volume 6 Issue 7, July 2017} www.ijsr.net 
$\leq\left\|z-x_{2 n}\right\|^{2}$

$$
\begin{aligned}
& +\alpha \frac{P_{\alpha}^{2}(z, T(z))+P_{\alpha}^{2}\left(x_{2 n-1}, S\left(x_{2 n-1}\right)\right)+P_{\alpha}^{2}\left(z, S\left(x_{2 n-1}\right)\right)}{1+P_{\alpha}^{2}\left(z, S\left(x_{2 n-1}\right)\right) P_{\alpha}^{2}\left(x_{2 n-1}, T(z)\right)} \\
& + \\
& \beta\left[P_{\alpha}^{2}(z, T(z))+P_{\alpha}^{2}\left(z, S\left(x_{2 n-1}\right)\right)\right] \\
& \leq\left\|z-x_{2 n}\right\|^{2} \\
& +\gamma\left\|x_{2 n-1}-z\right\|^{2} \\
& +\alpha \frac{P_{\alpha}^{2}(z, T(z))+\left\|x_{2 n-1}-x_{2 n}\right\|^{2}+\left\|z-x_{2 n}\right\|^{2}}{1+\left\|z-x_{2 n}\right\|^{2} P_{\alpha}^{2}\left(x_{2 n-1}, T(z)\right)} \\
& +\beta\left[P_{\alpha}^{2}(z, T(z))+\left\|z-x_{2 n}\right\|^{2}\right]+\gamma\left\|x_{2 n-1}-z\right\|^{2}
\end{aligned}
$$

$$
P_{\alpha}^{2}(z, T(z)) \leq \frac{\alpha}{1-\alpha-\beta}\left\|x_{2 n-1}-x_{2 n}\right\|^{2}
$$

Putting $q=\frac{\alpha}{1-\alpha-\beta}<1$

Then, we have

$$
P_{\alpha}^{2}(z, T(z)) \leq q\left\|x_{2 n-1}-x_{2 n}\right\|^{2} \leq q^{n}\left\|x_{2 n-1}-x_{2 n}\right\|^{2}
$$

As $\rightarrow \infty$, then $P_{\alpha}^{2}(z, T(z))=0$

Hence we get $\{z\} \subset T(z)$.

Similarly, $\{z\} \subset S(z)$.

\section{References}

[1] Jagdish C. Chaudhary, Jatin S. Patel, Shailesh T. Patel, Chirag R. Patel, "Some Common Fixed Point Theorems in Fuzzy Mappings" International Journal of Mathematics Trends and Technology- Volume20 Number2-April 2015

[2] L.A.zadeh, probability measures of fuzzy events, J.Math.Anal.Appl.23(1968), 421-427

[3] S.Heilpern, Fuzzy mappings and fixed point theorem, J.Math. Anal. 83 (1981), 566-569.

[4] S.B.Nadler, Multi valued contraction mappings, pacific J.Math. 30(1969), 475-488. 